\title{
Perioperative Practices and Outcome of Neurosurgery during the COVID-19 Pandemic: Institutional Experience and Retrospective Observational Analysis
}

\author{
Priyanka Khurana ${ }^{1}$ \\ Monica S. Tandon ${ }^{1}$ \\ Pragati Ganjoo, \\ Daljit Singh ${ }^{2}$ Anita Jagetia ${ }^{2}$
}

\begin{abstract}
Address for correspondence Pragati Ganjoo, DA DNB, Department of Anaesthesiology and Intensive Care, G.B. Pant Institute of Postgraduate Medical Education and Research, Jawahar Lal Nehru Marg, New Delhi 110002, India (e-mail: pganjoo@gmail.com).
\end{abstract}

Indian J Neurosurg 2021;10:128-135.

\section{Abstract \\ Keywords \\ - perioperative proto- cols in COVID-19 \\ - neurosurgery prac- tices and outcome \\ - health care worker infection}

Introduction Surgery during the coronavirus disease 2019 (COVID-19) pandemic is a major concern due to possibility of infection transmission among health care workers (HCWs) and patients, and a worsened surgical outcome; most surgeries are thus being deferred. However, we continued with emergency neurosurgeries using our own Neurosurgical Standard Operating Procedures (NS-SOPs). We describe here our institutional neurosurgical experience and observations of a retrospective analysis done to determine the incidence of workplace-acquired COVID infection among the HCWs, and the outcome of neurosurgery performed during the early phase of the on-going pandemic.

Methods Our NS-SOPs included a Screening Proforma, and protocols for the conduct of neurosurgery, starting from the preoperative period till death or postdischarge follow-up of the patients. Protocols to ensure safety and mental well-being of the HCWs were also implemented. Patient and HCW data from April 1 to August 31, 2020 was collected and analyzed for the postsurgical patient outcome and for determining the level of workplace-transmitted COVID infection.

Results Neurosurgeries were performed on 169 patients during this 5 -month period. We observed a cumulative mortality of $17 / 169(10.1 \%)$, with 5 patients having unexplained postoperative respiratory manifestations and rapid deterioration suggestive of COVID illness. Nineteen HCWs (8.83\%), mostly nurses, were infected, but only 3 (16.7\%) had workplace-acquired infection. The infections were sporadic with no cluster of infections observed.

Conclusion Implementation of standard perioperative protocols and their continuous scrutiny, evaluation, and modification is important to contain infection in HCWs and to improve the neurosurgical outcome during this pandemic. published online April 30, 2021
DOI https://doi.org/

$10.1055 / \mathrm{s}-0041-1729463$ ISSN 2277-954X (c) 2021. Neurological Surgeons' Society of India.

This is an open access article published by Thieme under the terms of the Creative Commons Attribution-NonDerivative-NonCommercial-License, permitting copying and reproduction so long as the original work is given appropriate credit. Contents may not be used for commercial purposes, or adapted, remixed, transformed or built upon. (https://creativecommons.org/licenses/by-nc-nd/4.0/).

Thieme Medical and Scientific Publishers Pvt. Ltd. A-12, 2nd Floor, Sector 2, Noida-201301 UP, India 


\section{Introduction}

Coronavirus disease 2019 (COVID-19) is a life-threatening illness caused by severe acute respiratory syndrome coronavirus 2 (SARS-CoV-2) virus that manifests as fever, cough, and breathlessness, but may rapidly progress to pneumonia, respiratory failure, septic shock, multiorgan dysfunction, and death; cardiac, gastrointestinal, dermatological, and neurological manifestations are also reported. ${ }^{1}$ Due to its rapid human-to-human transmission via direct aerosols dispersion or fomites, COVID-19 has now evolved into a pandemic that has crippled the entire world. Most hospitals, including ours, have deferred the management of chronic ailments and elective surgeries; however, we continued to undertake emergency neurosurgeries due to the devastating consequences of delaying them. ${ }^{2}$

Conducting neurosurgery during the COVID pandemic raises important concerns related to its risks and outcome. These include, the dreaded risk of transmitting this highly contagious disease among the health care workers (HCWs) of neurosurgical wards, operating theaters (OTs), and intensive care unit(ICU), and the possible perioperative deterioration of the surgical patient due to either, a newly acquired infection, or worsening of an existing infection; a 20 to $27 \%$ increase in critical postoperative complications and mortality has been reported in COVID positive surgical patients. ${ }^{3,4}$ Further, neurosurgical patients may be misdiagnosed with COVID illness due to overlapping respiratory symptoms, or COVID patients may be mistaken to have neurological disease due to manifestations like altered sensorium, seizures, unsteady gait, stroke, cognitive impairment, and localized neuralgia; the resultant mismanagement of both these conditions can be disastrous., ${ }^{5,6}$ A good knowledge of COVID illness and its perioperative implications in neurosurgery, and formulation of workplace protocols that address the safety concerns of HCWs and surgical patients is thus necessary; medical literature including perioperative management guidelines for this novel disease are still limited. ${ }^{7-12}$ We have devised our own Neurosurgical Standard Operating Procedures (NS-SOPs) for preventing infection transmission among the HCWs, and promptly managing any postoperative COVID-related worsening of neurosurgical patients (-Table 1 ). We describe here our institutional experience in the perioperative management of neurosurgical patients using these NS-SOPs and present observations from a retrospective study undertaken by us to evaluate, (1) the incidence of workplace-acquired COVID infection among the neurosurgical HCWs and (2) the outcome of neurosurgery done at our hospital during this pandemic.

\section{Methods}

From April 1, 2020 onwards, all patients admitted for emergency/semi-emergency neurosurgeries were managed using the NS-SOPs (-Table 1). The preoperative protocol included evaluation of neurological disease and triaging to determine surgical priorities, and screening of patients for COVID infection using a COVID Screening Proforma devised by us ( - Table 2 ). COVID suspects were identified based on well-defined criteria and confirmation was done using the real-time reverse transcriptase-polymerase chain reaction (RT-PCR) and TrueNat tests, as per the prevailing government guidelines. However, due to nonavailability of our own testing facilities and delayed test results from elsewhere, COVID suspects requiring emergent surgery were operated upon without testing, considering them as COVID positives. ${ }^{13}$ Mid-June onwards, all COVID suspects were tested in-house and confirmed positives identified. The risk-benefit ratio for neurosurgery in COVID positives was ascertained by comparing the hazards of operating during a pandemic with the adverse consequences of deferment ${ }^{14}$; thus, surgery was undertaken either immediately, or after the patients recovered in a COVID center. The intraoperative protocol included modifications in neurosurgical and neuroanesthesia practices to prevent infection transmission, especially avoiding the aerosol-generating procedures (AGPs). ${ }^{15}$ These intraoperative SOPs were also followed in COVID negatives as false negative RT-PCR results have been reported in up to 30 to $50 \%$ of tested patients. ${ }^{16}$ The postoperative protocol included close patient monitoring for newly developed or worsening features of COVID illness like fever, breathlessness, hypoxia, secondary pulmonary infection, multisystem involvement, and unexplained clinical deterioration and death; RT-PCR testing, X-ray chest, and computed tomography (CT) scan chest were done promptly for confirmation. Patients discharged from hospital were advised isolation at home for 10 days. They were contacted on phone after 2 weeks for any development of COVID-related manifestations, and if confirmed, were referred to a COVID center.

The NS-SOPs also included specific measures for enhancing the physical and mental well-being of our overstressed HCWs. Counseling and motivational sessions were conducted, and lifestyle changes and home safety measures advised (-Table 1). The HCWs were diligently monitored for development of COVID illness, and those testing positive, were isolated and referred to COVID centers. Aggressive contact tracing of COVID positives was done to determine the source of infection.

The data of the initial 5-month period was collected and analyzed. This included preoperative patient profile and clinical condition; COVID screening and testing results; neurosurgical details; perioperative course and complications; postdischarge follow-up of the patients; occurrences of COVID infection in HCWs; postinfection course; details of contact tracing; instances of breach in compliance of NS-SOPs; and number of unmotivated, careless, or frequently absent HCWs.

\section{Results}

Between April 1 and August 31,2020,175 patients were admitted for various neurosurgical emergencies/semi-emergencies in our hospital. The surgeries were triaged as emergent, if needed within 2 days, and urgent, if could be deferred for 2 weeks. ${ }^{14}$-Table 3 depicts the demographic profile and preoperative COVID-specific data of the admitted patients. Patients at high risk for developing COVID infection included 
Table 1 Standard operating procedures for neurosurgical patients
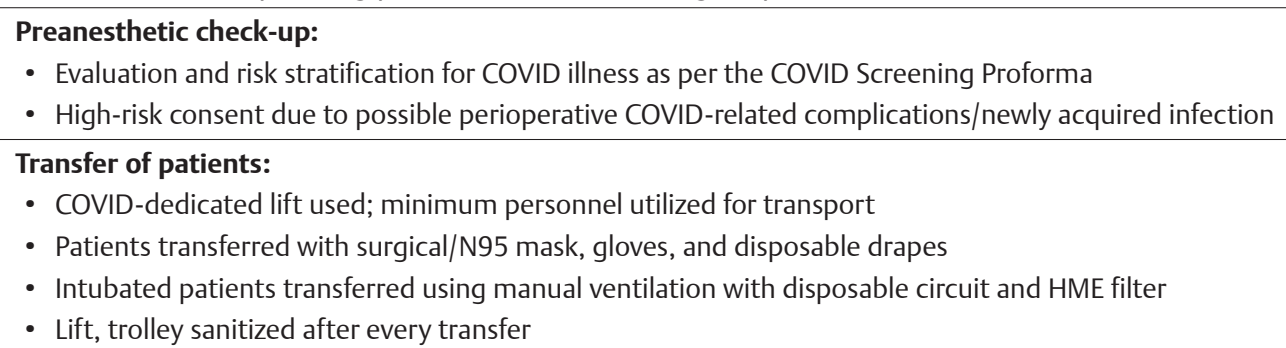

OT personnel:

- Staff to use PPE with N95 mask, face shield, water-resistant hooded gown, double gloves, shoe covers

- Regular training of HCWs for correct technique of PPE donning/doffing and reuse of N-95 masks

- Entry/exit from OT and shift changes restricted during surgery; Outside runner staff to assist inside staff

- Two teams of anesthetists and technicians, one only for airway management with no interchange allowed

OT preparation:

- OT fully prepared before patient transfer; Donning and doffing in designated areas with illustrations

- Minimum personnel and equipment inside OT; Maximum use of disposable items

- Two anesthesia trolleys inside OT, one for drugs, fluids, and other only for airway equipment

- Fill suction canister, dip scavenging port in $1 \%$ sodium hypochlorite

Conduct of surgery:

- Negative room pressure maintained, exhaust fans to keep running, split AC switched off before patient transfer and restarted 30 minutes after intubation; doors closed during surgery

- Patient positioning only by OT staff; Surgical team to enter OT 15 minutes after intubation/extubation

- AGPs to be avoided inside OT; All record keeping outside OT; minimize surgical time

- OT sanitization for 1 hour; Supervision by senior staff; Wear full PPE; Use disposable cleaning items; Discard airway disposables, dip other items in $1 \%$ hypochlorite for 30 minutes; Hydrogen peroxide fogging

- Waste disposal in bins with double bags, linen in sealed 2 bags; bins/trolleys sprayed with $1 \%$ hypochlorite

COVID-specific anesthesia protocol: Avoid anesthetic AGPs

- Avoid coughing; Use anti-sialagogues, antiemetics; Preoxygenate, RSI; Experienced intubating anesthetist

- Video-laryngoscope, intubation box; 2 HME filters on circuit; Closed suctioning; Postextubation mask

COVID-specific neurosurgical protocol: Avoid neurosurgical AGPs

- Bone drilling inside plastic cover; avoid Mayfield head clamp; use dedicated OT surgical markers

- Send specimen in tight-fit plastic box sealed inside plastic bag; Wear lead apron under PPE and disinfect

Postoperative management:

- Oxygen therapy, early DVT prophylaxis for all patients; no AGPs in ICU

- Close monitoring for early detection of COVID-related clinical deterioration, new-onset COVID illness; Urgent chest X-ray and CT scan available; low threshold for intubation/ ventilation

- Restricted attendant entry in the ICU; Patient discharge as per prevailing government guidelines

- Postdischarge telephonic follow-up of patients

Lifestyle changes and home safety measures for HCWs:

- Ensure good personal hygiene, balanced diet and 8 hours sleep

- Avoid belts, wallets, cash inside OT, minimize cellphone use; clean with $70 \%$ alcohol

- Avoid keeping beard to allow proper N-95 mask seal

- Change footwear before entering home; Leave keys/wallet in a separate box at entrance

- Frequent hand hygiene, shower, change of clothes; separate linen and utensils at home

- Maintain social distancing from family members

- Curtail/quit smoking, manage comorbidities like diabetes, hypertension

Abbreviations: AGPs, aerosol-generating procedures; CT, computed tomography; DVT, deep vein thrombosis; HCWs, health care workers; HME, heat moisture exchanger; HP, histopathological; ICU, intensive care unit; OT, operating theater; PPE, personal protective equipment; RSI, rapid sequence induction.

those above 60 years (11 patients; 6.3\%), below 1 year (18 patients; $10.5 \%$ ), and having comorbidities (102 patients; $58.3 \%$ ). COVID screening detected 48 patients (27.6\%) as COVID suspects, of which 8 patients could not be tested due to their emergent surgery and were considered as COVID positives.
Testing in the remaining 40 COVID suspects revealed 9 COVID positives who were shifted to a COVID center to recover. While 3 patients underwent surgery after testing negative, 6 patients did not report back; the 31 negatives out of the 40 COVID suspects also underwent subsequent surgery. The 
Table 2 COVID screening proforma for neurosurgical patients (salient features)

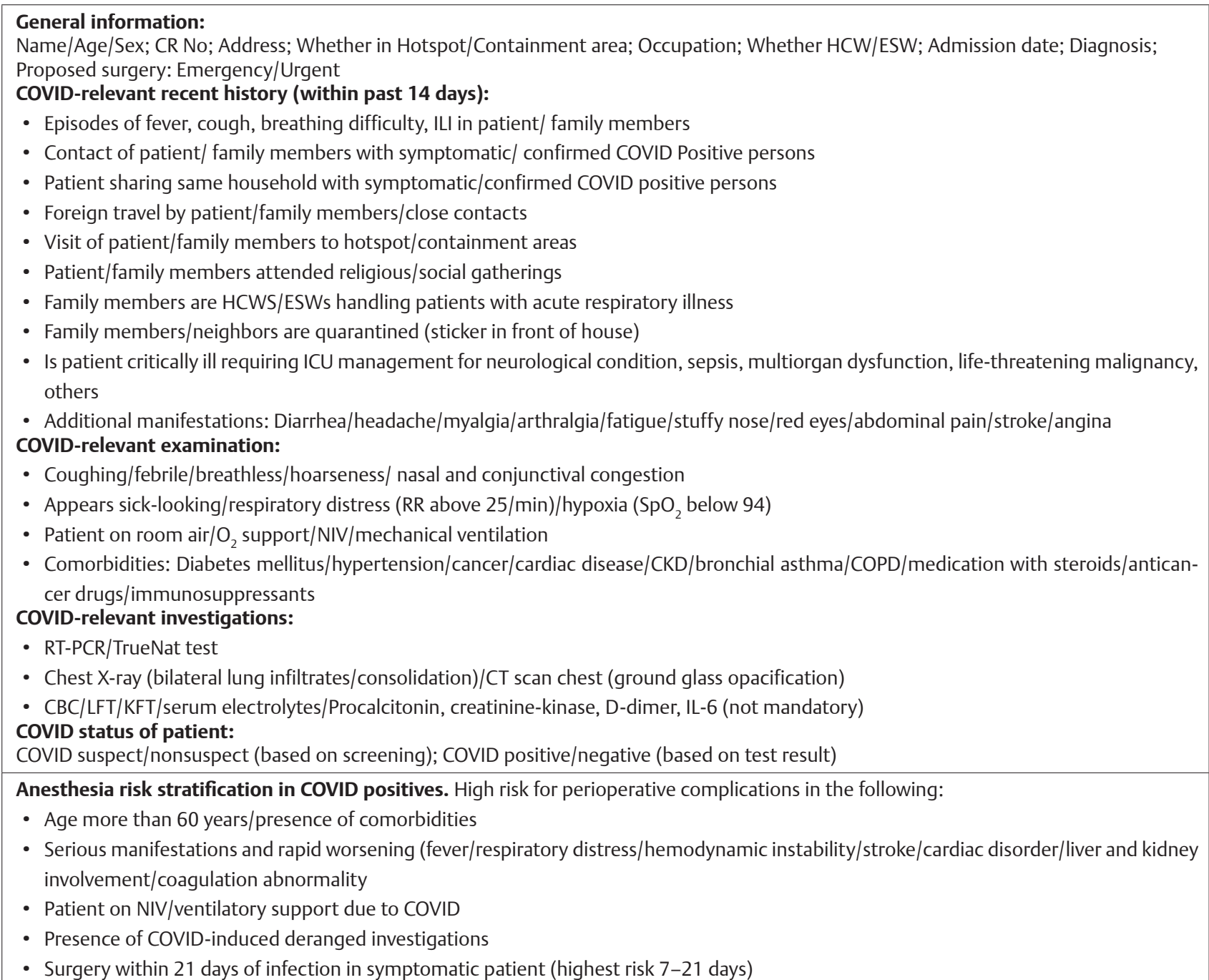

Abbreviations: CKD, chronic kidney disease; COPD, chronic obstructive pulmonary disease; CT, computed tomography; CBC, complete blood count; ESW, essential services worker; HCW, health care worker; IL-6, interleukin 6; ILI, influenza-like illness; KFT, kidney function test; LFT, liver function test; NIV, noninvasive ventilation; $\mathrm{RR}$, respiratory rate; $\mathrm{SpO}_{2}$, oxygen saturation of blood.

127 COVID nonsuspects on screening were not tested as per the prevailing government guidelines and were considered as COVID negatives for surgery. In all, 169 neurosurgeries were undertaken at our institution during this period ( - Table 4 ).

\section{Workplace-Acquired COVID Infection among Our Neurosurgical HCWs}

Out of the 215 HCWs in neurosurgery and neuroanesthesia, 19 developed clinical features resembling COVID illness like cough $(17 ; 89.5 \%)$, fever $(7 ; 36.8 \%)$, myalgia $(4 ; 21.1 \%)$, and breathlessness (2; 10.5\%). Testing detected 19 HCWs (8.83\%) to be COVID positive, out of which 10 (53\%) were nurses, 4 (21\%) were OT technicians, 2 (10\%) were doctors, and 3 (16\%) were nursing orderlies and sweepers (-Fig. 1A). Contact tracing revealed that $1158 \%$ HCWs acquired the infection from external sources like infected family members/neighbors or from attending social/religious gatherings. The source was uncertain in $5(26 \%)$ HCWs. The infection was clearly workplace-acquired in $3(16 \%)$ nurses who worked and lunched together; they developed symptoms sequentially within 2 to 3 days after the first sick nurse got infected from a family member ( - Fig. 1B). Infections at our hospital were sporadic with no cluster of infections observed at any time. The infected HCWs were mostly home-quarantined, but 2 of them were shifted to a COVID center after developing breathlessness and hypoxia; all the HCWs returned to work after full recovery. There were approximately 20 instances of carelessness in adhering to the NS-SOPs but no report of any unmotivated or frequently absenting HCWs.

\section{Course and Outcome of Neurosurgery at Our Hospital during the COVID Pandemic}

Among the 8 COVID suspects considered as positives for surgery, 1 patient developed fever and pneumonia-like features on the second postoperative day (POD), and his CT scan chest showed bilateral lower-lobe ground-glass opacities typical of COVID infection; but he died without getting COVID tested. Another patient on ventilator developed unexpected hypoxia and extensive bilateral lung infiltrates on CT scan suggestive of COVID and died; he had tested negative. Among the 31 COVID negatives, 3 patients died after surgery due to 

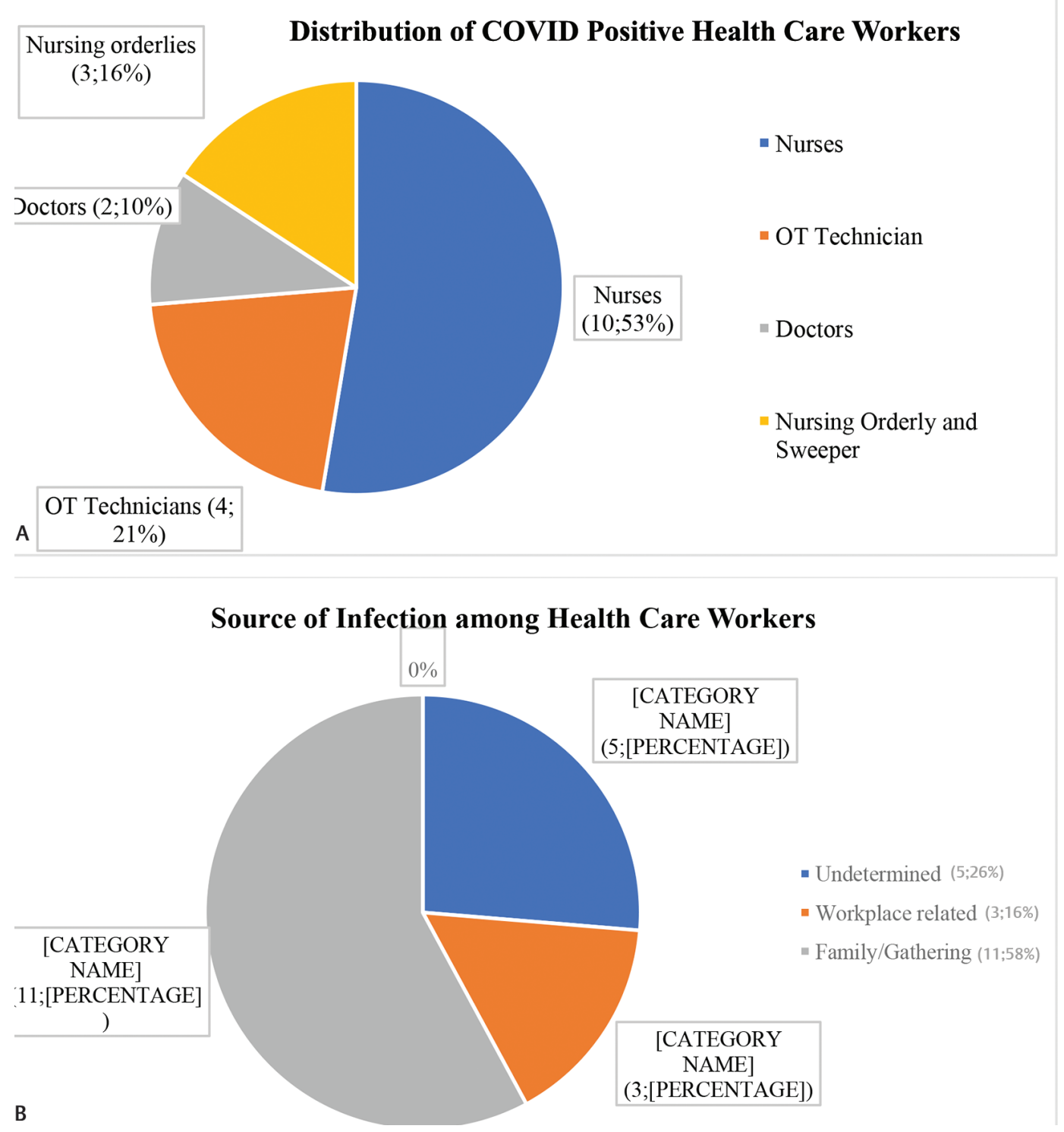

Fig. 1 Coronavirus disease 2019 (COVID-19)infection among Health Care Workers. (A) Distribution of COVID positive health care workers. (B) Source of infection among health care workers.

neurosurgical complications, but 1 patient developed sudden, unexplained clinical deterioration on the first POD and died before COVID testing. Out of the 127 COVID nonsuspects who were not tested before surgery, 9 patients died from identifiable neurosurgical complications, but 2 patients had unexpected early death, of which, 1 patient had clinical and radiographic features of acute respiratory distress syndrome (ARDS). The overall perioperative mortality rate was $10.1 \%$ (17/169 patients); mortality in COVID suspects was $12.5 \%$ (6/48 patients) and in COVID nonsuspects was 8.6\% (11/127 patients). Out of the total 17 deaths, 5 postoperative deaths were unexpected and quick; their cause, though strongly suspected to be due to COVID illness by the typical clinical manifestations, could not be ascertained in the absence of confirmatory testing. None of the discharged patients developed COVID illness within 2 weeks of leaving hospital. Our observations are summarized as a flowchart in (-Fig. 2).

\section{Discussion}

The HCWs are at high risk of developing COVID illness due to their proximity to infected patients and involvement in AGPs; a 12 time increased risk of testing positive is reported in frontline HCWs. ${ }^{17}$ The neurosurgical HCWs are particularly vulnerable due to their increased handling of untested emergency patients, repeated intubations, prolonged ventilations, and frequent chest physiotherapies. COVID-specific teaching and training programs and implementation of precautionary measures at work are thus needed to ensure their safety during the pandemic. We dispersed medical literature and conducted hands-on workshops on COVID-related issues, particularly the preventive measures and correct donning/doffing methods. Perioperative NS-SOPs were devised and applied starting from admission to discharge/death of the patients (-Tables $\mathbf{1}$ and 2). AGPs in the OT and ICU were identified 
Table 3 Demographic profile of admitted neurosurgical patients $(n=175)$

\begin{tabular}{|c|c|}
\hline $\begin{array}{l}\text { Age }(y) \text {, mean } \pm \text { SD } \\
\text { Age, } n(\%) \\
\text { - Age }<1 \text { y } \\
\text { - Age }<18 y \\
\text { - Age } 18-60 y \\
\text { - Age }>60 y\end{array}$ & $\begin{array}{l}29.6 \pm 20.7 \\
18(10.5 \%) \\
61(34.8 \%) \\
85(48.6 \%) \\
11(6.3 \%)\end{array}$ \\
\hline Sex, $n$ (\%): Males; Females & $\begin{array}{l}99(56.8 \%) ; 76 \\
(43.4 \%)\end{array}$ \\
\hline $\begin{array}{l}\text { Patients with comorbidities, } n(\%) \\
\text { - Diabetes mellitus } \\
\text { - Hypertension } \\
\text { - Cardiac disease } \\
\text { - Chronic kidney disease } \\
\text { - COPD/bronchial asthma/pulmonary arte- } \\
\quad \text { rial hypertension }\end{array}$ & $\begin{array}{l}41(23.4 \%) \\
29(16.6 \%) \\
6(3.4 \%) \\
11(6.3 \%) \\
15(8.6 \%)\end{array}$ \\
\hline $\begin{array}{l}\text { Patients with COVID status on screening, } n(\%) \\
\text { - COVID suspects } \\
\text { - COVID nonsuspects }\end{array}$ & $\begin{array}{l}48(27.4 \%) \\
127(72.6 \%)\end{array}$ \\
\hline $\begin{array}{l}\text { COVID suspects as per screening criteria, } n \text { (\%) } \\
\text { - Residing in hotspots/containment zones } \\
\text { - HCWs, ESWs } \\
\text { - Self-symptomatic } \\
\text { - Symptomatic close contacts } \\
\text { - Attended social/religious gatherings }\end{array}$ & $\begin{array}{l}26(14.7 \%) \\
4(2.2 \%) \\
7(3.2 \%) \\
7(3.2 \%) \\
4(1.1 \%)\end{array}$ \\
\hline $\begin{array}{l}\text { Testing status of } 48 \text { COVID suspects, } n(\%) \text { : } \\
\text { - Patients couldn't be tested before surgery } \\
\text { - Patients tested before surgery } \\
\text { - COVID positives } \\
\text { - COVID negatives }\end{array}$ & $\begin{array}{l}8(3.2 \%) \\
40(28.6 \%) \\
9(8.6 \%) \\
31(16.8 \%)\end{array}$ \\
\hline
\end{tabular}

Abbreviations: COPD, chronic obstructive pulmonary disease; ESWs, essential services workers; HCWs, health care workers; SD, standard deviation.

Table 4 Neurosurgeries undertaken at our institute during the initial 5 months

\begin{tabular}{|l|l|}
\hline Aneurysmal surgeries & $29(17.1 \%)$ \\
- Craniotomy and clipping & $9(5.3 \%)$ \\
- Endovascular coiling & $20(11.8 \%)$ \\
\hline Craniotomy and tumor excision & $66(39.1 \%)$ \\
- Meningiomas & $26(15.4 \%)$ \\
- Glioblastomas & $14(8.2 \%)$ \\
- Situitary adenomas & $7(4.1 \%)$ \\
- Pineal gland tumors & $6(3.5 \%)$ \\
- Metastatic brain tumors & $2(1.1 \%)$ \\
\hline Surgeries for hydrocephalous & $1(0.6 \%)$ \\
- Primary V-P shunting & $46(27.2 \%)$ \\
- Revision V-P shunting & $12(7.1 \%)$ \\
- ETV & $16(9.5 \%)$ \\
\hline Burr-hole surgeries & $18(10.7 \%)$ \\
\hline Spine surgeries & $6(3.5 \%)$ \\
\hline Decompressive craniotomies & $7(4.1 \%)$ \\
\hline Abbreviations: ETV, endoscopic third ventriculostomy; VP, \\
ventriculo-peritoneal.
\end{tabular}

and avoided. These included bag-mask ventilation, awake intubation, bronchoscopy, tracheostomy, nebulization, noninvasive ventilation, high-flow nasal oxygen delivery, chest physiotherapy, open suctioning, open bone-drilling, use of cavitron ultrasonic surgical aspirator, awake craniotomies, and endoscopic procedures; ${ }^{10,11,18}$ emergency transsphenoidal hypophysectomy has been shown to cause viral transmission to multiple HCWs even with appropriate personal protective equipment (PPE). ${ }^{18}$ Specific modifications were made in our routine neurosurgical practice like using OTs with high air change frequencies and particulate air filters ${ }^{7}$ and use of intubation boxes, performing bone drilling with low-speed electric drills inside a plastic cover and with copious irrigation, use of electrocautery at low power, and preventing sinus breach during surgery. ${ }^{11}$ We preferred endovascular coiling (11.8\%) over aneurysm clipping (5.3\%) and endoscopic third ventriculostomy (10.7\%) over ventriculoperitoneal shunt surgery (7.1\%) and avoided resection of pituitary adenomas through the transsphenoidal route and instrumentation of spine. Delaying of definitive surgeries, advising palliative care, performing emergency procedures under local anesthesia, and use of alternate treatments like external ventricular drainage, antiedema measures, and radiosurgery for brain tumors are further neurosurgical recommendations. ${ }^{10,12,14,15,18}$ HCWs on duty during this COVID pandemic are facing immense psychological stress due to the fear of getting infected, the challenges of remembering and strictly following the evolving SOPs, enduring the discomfort of working in PPEs, extended working hours, and the anxiety of carrying the infection to their homes. To cope with these worries, our HCWs were counseled repeatedly and encouraged to adopt the hospital and home lifestyle changes incorporated in our NS-SOPs ( - Table $\mathbf{1}$ ).

Prior literature on neuroanesthesia and neurosurgery SOPs is available, though limited. ${ }^{7-12}$ Few authors have evaluated the impact of SOPs in preventing workplace-acquired COVID infection among the HCWs, like done in this study. The incidence of infection among our HCWs was $8.83 \%$ in the 5 -month period under study, and only sporadic instances were found. Alajmi et al reported a 10.6\% incidence of COVID infection among their HCWs; most HCWs acquired the infection at a non-COVID facility like ours. ${ }^{19}$ In another study, $9.8 \%$ of HCWs had prevalence of antibodies for SARS-CoV-2 infection during a 14-day period..$^{20}$ Most of our COVID positive HCWs attributed their infection to an outside source with only 3 out of 19 (15.7\%) instances of workplace-acquired infection. A similar incidence (16.7\%) was reported from Singapore during the initial period of pandemic, ${ }^{21}$ and from Netherland, where no in-hospital transmission was observed, and most instances were acquired from a community outbreak ${ }^{22}$; the risk of HCWs acquiring infection from an outside source was seen to rise significantly with increased community transmission. ${ }^{23}$ We found the highest infection among staff nurses (53\%) and least among doctors (10\%); similar observations were also reported previously. ${ }^{24}$ To prevent future instances of HCWs infection we imposed stricter social distancing and sanitizing norms and ensured stringent compliance with NS-SOPs.

COVID-infected patients needing emergent surgical intervention were found to have poorer outcomes compared with their matched controls. In a study conducted among various surgical disciplines, including neurosurgery, a significantly higher mortality was reported in the COVID-19 group compared with the control group (8 patients: $19.51 \%$ vs. 


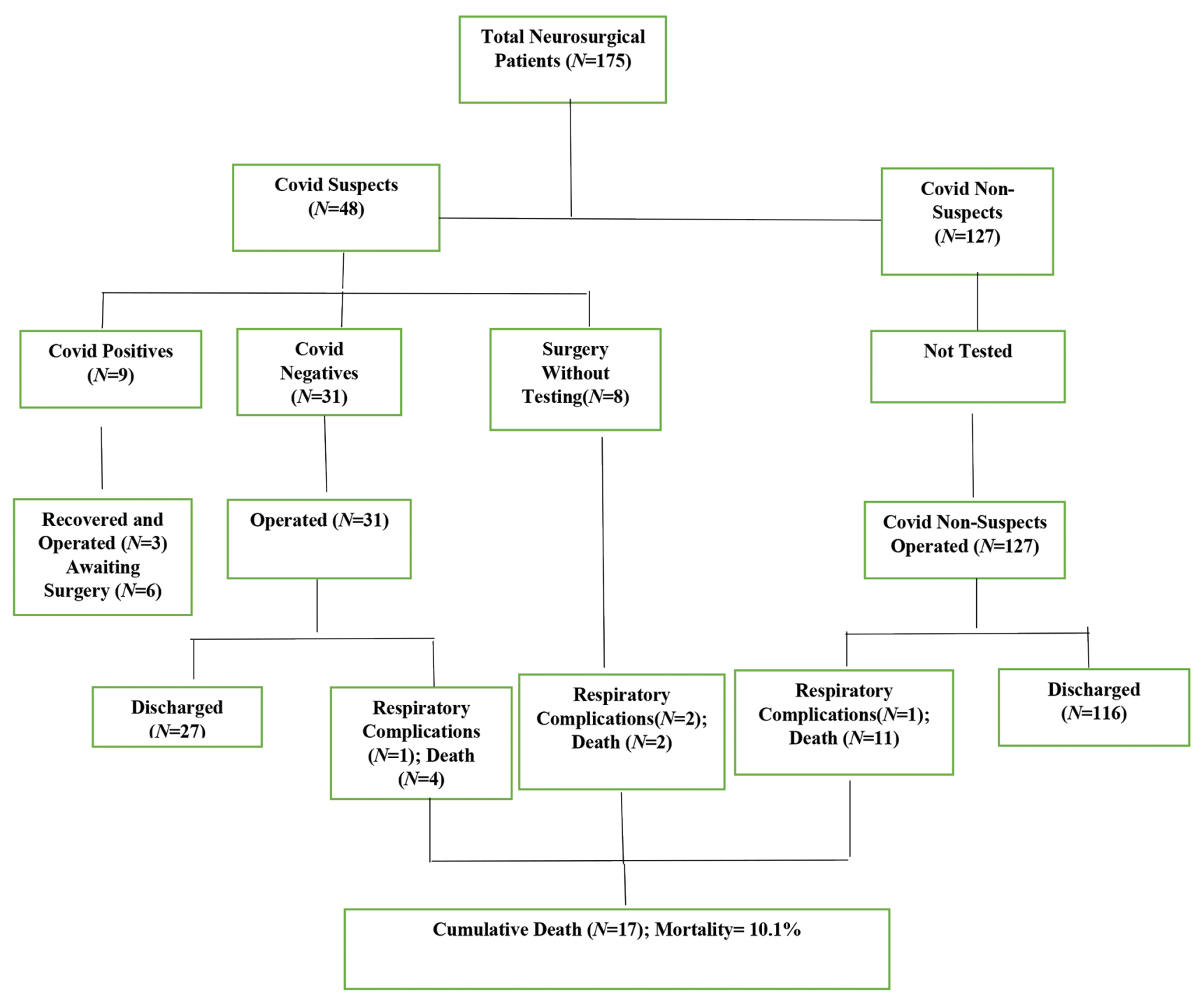

Fig. 2 Flowchart depicting the course and outcome of the study.

2 patients: $2.44 \%$; odds ratio 9.5 ; $95 \%$ confidence interval, 1.77-96.53). ${ }^{25}$ Other authors reported surgical patients who acquired perioperative COVID infection to have an overall 30-day mortality of $23.8 \%$ (268 of 1,128 patients), with a high incidence of pulmonary complications (577 patients: 51.2\%). ${ }^{26}$ The high mortality in COVID-infected patients is attributed to inflammatory mediators causing lung injury and ARDS, and this may be more pronounced in neurosurgical patients with preexisting pulmonary complications ${ }^{27}$; the resultant hypoxia and hemodynamic instability may jeopardize cerebral oxygenation and worsen the outcome of neurosurgery. ${ }^{28}$ We observed postoperative development of unexplained severe respiratory complications strongly suggestive of COVID illness that resulted in the death of 5 patients. The fact that one of these patients was COVID negative and 2 patients were nonsuspects reiterates that screening and testing may not be sufficient to identify patients infected with SARS-CoV-2 virus. ${ }^{16}$ We kept a high level of suspicion for COVID throughout the perioperative period, and on appearance of early signs and symptoms, the patients were immediately isolated and treated. These measures are important to contain the mortality and further spread of the virus.

\section{Conclusion}

Thus, implementing distinct perioperative protocols in neurosurgery is essential to minimize the adverse impact of COVID illness on staff and patients, as was experienced by us. Preoperative screening, intraoperative avoidance of AGPs, vigilant postoperative monitoring, close postdischarge patient follow-up, and surveillance of HCWs were the highlights of our NS-SOPs. This was the initial evaluation of our neurosurgical protocols and practices. With progression of the pandemic and added knowledge of the COVID illness, frequent reassessments and modifications of such protocols is required.

\section{Funding}

None.

\section{Conflict of Interest}

None declared. 


\section{References}

1 Vetter P, Vu DL, L'Huillier AG, Schibler M, Kaiser L, Jacquerioz F. Clinical features of COVID-19. BMJ 2020;369:m1470

2 Tsermoulas G, Zisakis A, Flint G, Belli A. Challenges to neurosurgery during the coronavirus disease 2019 (COVID-19) pandemic. World Neurosurg 2020;139:519-525

3 Lei S, Jiang F, Su W, et al. Clinical characteristics and outcomes of patients undergoing surgeries during the incubation period of COVID-19 infection. EClinicalMedicine 2020;21:100331

4 Nahshon C, Bitterman A, Haddad R, Hazzan D, Lavie O. Hazardous postoperative outcomes of unexpected COVID-19 infected patients: a call for global consideration of sampling all asymptomatic patients before surgical treatment. World J Surg 2020;44(8):2477-2481

5 Khatoon F, Prasad K, Kumar V. Neurological manifestations of COVID-19: available evidences and a new paradigm. J Neurovirol 2020;26(5):619-630

6 Ellul MA, Benjamin L, Singh B, et al. Neurological associations of COVID-19. Lancet Neurol 2020;19(9):767-783

7 Malhotra N, Bajwa SJ, Joshi M, Mehdiratta L, Trikha A. COVID operation theatre- advisory and position statement of Indian Society of Anaesthesiologists (ISA National) Indian J Anaesth 2020;64(5):355-362

8 Nedunchezhian AS, Ajayan N, P APH, Prathapadas U, Sethuraman M, Koshy T. Finding the calm in the chaos: an institutional protocol for anesthetic management of a patient for neurosurgery during coronavirus disease 2019 pandemic. J Neurosci Rural Pract 2020;11(3):369-374

9 Singh SK, Abhijit V, Jha VC. Modification of neurosurgical practice during corona pandemic: our experience at AIIMS Patna and long term guidelines. Interdiscip Neurosurg 2021;23:100895

10 Thapa A, Sharma K, Shrestha P. Standard operating protocol: operating on COVID-19 patients. Nepal J Neurosci 2020;17(1):66-71

11 Coccolini F, Perrone G, Chiarugi M, et al. Surgery in COVID-19 patients: operational directives. World J Emerg Surg 2020;15(1):25

12 Muhammad S, Tanikawa R, Lawton MT, Niemelä M, Hänggi D. Letter: safety instructions for neurosurgeons during COVID-19 pandemic based on recent knowledge and experience. Neurosurgery 2020;87(2):E220-E221

13 Srinivasaiah B, Deora H. Anesthetic management of neurosurgery patients in COVID-19 pandemic in an emergency setting. J Neurosci Rural Pract 2020;11(4):514-516

14 Burke JF, Chan AK, Mummaneni V, et al. Letter: the coronavirus disease 2019 global pandemic: a neurosurgical treatment algorithm. Neurosurgery 2020;87(1):E50-E56

15 Gupta P, Muthukumar N, Rajshekhar V, et al. Neurosurgery and neurology practices during the novel
COVID-19 pandemic: a consensus statement from India. Neurol India 2020;68(2):246-254

16 Yang Y, Yang M, Shen C, et al. Evaluating the accuracy of different respiratory specimens in the laboratory diagnosis and monitoring the viral shedding of $2019-\mathrm{nCoV}$ infections. The Innovation. 2020;1(3)10.1016/j.xinn.2020.10006

17 Nguyen LH, Drew DA, Joshi AD, et al. Risk of COVID-19 among frontline healthcare workers and the general community: a prospective cohort study. medRxiv 2020. Doi: $10.1101 / 2020.04 .29 .20084111$

18 Flexman AM, Abcejo AS, Avitsian R, et al. Neuroanesthesia practice during the COVID-19 pandemic: recommendations from Society for Neuroscience in Anesthesiology and Critical Care (SNACC) J Neurosurg Anesthesiol 2020;32(3):202-209

19 Alajmi J, Jeremijenko AM, Abraham JC, et al. COVID-19 infection among healthcare workers in a national healthcare system: the Qatar experience. Int J Infect Dis 2020;100:386-389

20 Jeremias A, Nguyen J, Levine J, et al. Prevalence of SARS-CoV-2 infection among health care workers in a tertiary community hospital. JAMA Intern Med 2020;e204214: DOI: $10.1001 /$ jamainternmed.2020.4214

21 Wong LY, Tan AL, Leo YS, Lee VJM, Toh MPHS. Healthcare workers in Singapore infected with COVID-19: 23 January-17 April 2020. Influenza Other Respir Viruses 2020. 10.1111/irv.12803

22 Kluytmans-van den Bergh MFQ Buiting AGM, Pas SD, et al. Prevalence and clinical presentation of health care workers with symptoms of coronavirus disease 2019 in 2 Dutch hospitals during an early phase of the pandemic. JAMA Netw Open 2020;3(5):e209673

23 Muhi S, Irving LB, Buising KL. COVID-19 in Australian health care workers: early experience of the Royal Melbourne Hospital emphasises the importance of community acquisition. Med J Aust 2020;213(1):44-44.e1, e1

24 Zheng L, Wang X, Zhou C, et al. Analysis of the infection status of healthcare workers in Wuhan during the COVID-19 outbreak: a cross-sectional study. Clin Infect Dis 2020;71(16):2109-2113

25 Doglietto F, Vezzoli M, Gheza F, et al. Factors associated with surgical mortality and complications among patients with and without coronavirus disease 2019 (COVID-19) in Italy. JAMA Surg 2020;155(8):1-14

26 COVIDSurg Collaborative. Mortality and pulmonary complications in patients undergoing surgery with perioperative SARS-CoV-2 infection: an international cohort study. Lancet 2020;396(10243):27-38

27 Guleria R, Madan K. Pulmonary complications in neurosurgical patient. Indian J of Neurosurg 2012;1:175-180

28 Lin CM, Lin MC, Huang SJ, et al. A prospective randomized study of brain tissue oxygen pressure-guided management in moderate and severe traumatic brain injury patients. BioMed Res Int 2015;2015:529580 